Originally published as:

Dazzi, A., Deniset-Besseau, A., Lasch, P.

Minimising contributions from scattering in infrared spectra by means of an integrating sphere (2013) Analyst, 138 (14), pp. 4191-4201.

DOI: $10.1039 / \mathrm{c} 3 \mathrm{an} 00381 \mathrm{~g}$

This is an author manuscript.

The definitive version is available at: http://pubs.rsc.org/ 


\title{
Minimising Contributions from Scattering in Infrared Spectra by Means of an Integrating Sphere
}

\author{
Alexandre Dazzi ${ }^{1}$, Ariane Deniset-Besseau ${ }^{1}$, Peter Lasch ${ }^{2, \$}$ \\ ${ }^{1}$ Laboratoire de Chimie Physique, UMR8000, Université Paris-Sud 11, 91405 Orsay, France. \\ ${ }^{2}$ Robert Koch-Institute, Centre for Biological Security 6 (ZBS 6), Nordufer 20, 13353 Berlin, \\ Germany
}

\$ corresponding author, e-mail: LaschP@rki.de

Keywords: Infrared spectroscopy, FT-IR, scattering, absorption, extinction, Mie scattering, resonant Mie scattering

Abbreviations: FTIR, Fourier-transform infrared; tt-FTIR, transmission-type FTIR; is-FTIR, integrating sphere FTIR; PMMA, poly(methyl methacrylate); DLaTGS, deuterated L-alanin doped triglycine sulfate; RMieS, Resonant Mie Scattering 


\section{Abstract}

Mid-infrared spectra of biological matter such as tissues, or microbial and eukaryotic cells measured in a transmission-type optical setup frequently show strongly distorted line shapes which arise from mixing of absorption and scattering contributions. Scattering-associated distorted line shapes may considerably complicate the analysis and interpretation of the infrared spectra and large efforts have been made to understand the mechanisms of scattering in biological matter and to compensate for spectral alterations caused by scattering. The goals of the present study were twofold: Firstly, to get a deeper understanding of the physics of scattering of biological systems and to explore how physical parameters of the scatterers such as shape, size and refractive index influence the line shape distortions observed. In this context, simulations based on the full Mie scattering formalism for spherical particles were found to be useful to explain the characteristics of the Mie scatter-associated distortions and yielded a size criterion for the scattering particles similar to the well-known near field criterion.

The second objective of the study was to investigate whether alternative optical setups allow to minimise the effects of scattering. For this purpose, an optical system is proposed which is composed of an integrating sphere unit originally designed for diffuse reflection measurements, an off-axis DLaTGS detector to collect scattered and transmitted light components and a commercial Fourier transform infrared (FTIR) spectrometer. In the context of this study transmission type (tt-) FTIR spectra and spectra acquired by means of the integrating sphere setup (is-FTIR) were acquired from monodisperse poly(methyl) methacrylate (PMMA) microspheres of systematically varying size. The tt-FTIR spectral data of different PMMA particles confirmed earlier observations such as the presence of size-dependent oscillating spectral baselines, peaks shifts, or derivative-like spectral line shapes. Such effects could be dramatically minimised when is-FTIR spectra were acquired by the integrating sphere unit. Utilisation of an integrating sphere is suggested as a convenient and easy-to use alternative to computer-based methods of scatter correction.

\section{Introduction}

The last fifteen years have seen significant progress in the field of applications of infrared (IR) spectroscopy. Advances in instrumentation such as the development of sensitive detectors ${ }^{1}$, coupling of IR microscopy and IR spectroscopy (IR microscopy) ${ }^{2,3}$ and the introduction of IR imaging systems with multi-channel focal plane array detectors ${ }^{4}$ have expanded the capabilities of IR spectroscopy and significantly opened up new fields of application of this methodology. Today, the areas of application of IR spectroscopy are manifold, ranging from classical analytical chemistry, art conservation, archaeology, forensics, pharmaceutics, microbiology and involve also medical research activities to develop application for histology, pathology and cytology. Concurrently with the numerous technical improvements in the field of IR spectroscopy, novel methods of spectral data analysis were introduced that helped to analyse the increasing amounts of data and to extract the relevant chemical and structural information. Examples of such methods are 
novel techniques for spectral pre-processing, ${ }^{5-9}$ multivariate spectral analysis (fingerprint techniques) ${ }^{10-11}$, and advanced segmentation methods for hyperspectral imaging. ${ }^{12-14}$

The expansion of the applications of IR spectroscopy, IR microscopy and IR imaging also showed the limits of the applicability of the techniques. Analysis of IR spectroscopic data is based on the assumption that the sample under study absorbs IR light of different wavelengths in a way that is characteristic for it. The absence of other types of optical processes (scattering) with an impact on the spectral characteristics of transmitted light is considered an important prerequisite for data interpretability and subsequent quantitative and qualitative spectral analyses. Typical measurement setups for IR absorption measurements are transmission, reflection, transflection and ATR-type configurations. All these measurement setups have in common that scattering as the second important optical process associated with the interaction of light and matter cannot be neglected. ${ }^{15}$ Many studies have shown that scattering may significantly alter the typical band profiles of IR absorption spectra. Because many biomedical applications of IR spectroscopy rely on observing specific disease-associated spectral markers or signatures, scattering renders the analysis of the spectral data very difficult if not impossible. ${ }^{7,8,15-18}$ IR spectral scattering effects are manifested in a typical manner and express themselves as altered spectral baselines (baseline slopes, undulating baselines), shifted peak positions, derivative-like band profiles, or may even lead to inverted (negative) peak shapes. ${ }^{15,17}$

Many authors have stated that spectra from biomedical samples such as cells and tissues can be influenced by factors other than the biochemical composition. ${ }^{18}$ For example, Mohlenhoff et al. reported that the nuclei of eukaryotic cells act as scatterers causing a broad, undulating spectral background onto which the absorption features are superimposed. ${ }^{7,16}$ The spectral baseline effects could be successfully modelled by the well-known Mie theory ${ }^{16,19,20}$ which applies to scattering of dielectric spheres of a size similar to the wavelength of light. Based on these findings Kohler et al. suggested a computational method to correct for the undulating baseline patterns. ${ }^{21}$ These models could, however, not explain the origin of band distortions like derivative-like spectral line shapes, band splitting or inverted IR bands sometimes mistakenly called the "dispersion artefact". ${ }^{22}$ In 2009, Bassan et al. demonstrated then that such band distortions are a result of both, scattering and the fact that the real part $n_{r}$ and the imaginary part $\kappa$ of the refractive index are linked via the Kramers-Kronig relationship. ${ }^{17}$ The variation of $n_{r}$ and $\kappa$ in the region of an absorption band (i.e. in resonance) prompted the authors to term this optical process Resonant Mie Scattering (RMieS). ${ }^{17}$ Based on this finding, the same group developed in the following years theoretical models of scattering which could be incorporated into computer algorithms to minimise unwanted scattering contributions from IR spectra showing both, broad Mie-type scattering features and spectral characteristics caused by RMieS. ${ }^{8,22}$

Although with the described computer models tremendous progress has been achieved, it must be noted that scattering effects cannot be always completely eliminated from the experimental data. This is to a large extent due to the fact that the theoretical models of Mie scattering basically apply to spherical objects. This precondition is, however, often not satisfied in the field of biomedical applications of IR spectroscopy, where objects with irregular morphology such as cells, tissues or microorganisms are studied. The assumption, that any non-spherical object can be approximated by 
a plurality of spheres having different radii certainly holds true. But one should always keep in mind that this is only a first order approximation and not an analytical solution. Another problem is the lack of knowledge about the exact values of parameters like the complex refractive index $n$, or the radii $r$ of the particles composing the sample. In the approach suggested by Bassan et al. ${ }^{8,22}$ this problem was addressed by simultaneously solving a multitude of equations for a set of spherical microparticles having different radii and refractive indices. For estimating the scattering contributions an iterative procedure was suggested that involves repetitively fitting the superposition of compressed solutions to a given reference spectrum. The suggested procedure is complex, computational demanding and often inaccessible to the practitioner. In addition, separation of scattered contributions and purely absorptive components in the measured spectra suffers also from the problem that a reference spectrum is required which must not contain any scattering contributions. Unfortunately, this condition cannot be always met.

With the present study we suggest an alternative solution to minimise contributions from scattering in the IR absorption spectra. The proposed approach is based on a radically different principle: instead of trying to eliminate scattering effects by computer-based computational methods, we propose to collect scattered light components by means of an integrating sphere unit which is equipped with an IR sensitive off-axis detector. The suggested optical configuration has been used to spectroscopically characterise strong IR scatterers of known chemical composition, poly(methyl methacrylate) (PMMA) beads. IR spectra acquired from PMMA microparticles of various radii were systematically analysed and compared with the respective spectra of a conventional transmission-type experiment.

\section{Theory of absorption and scattering}

\section{II.1 Absorption and scattering}

Absorption: Absorption and scattering are the two major optical processes describing the interaction of light with a given material. In optical spectroscopy absorption refers to the attenuation of light by which the energy of electromagnetic radiation is transformed into other forms. The extent of light attenuation by a given sample (see Fig. 1a) can be described by the well-known law of BeerLambert:

$$
\frac{I_{T}}{I_{0}}=10^{-\varepsilon C d}=10^{-A}
$$

In equation (1) $A$ is the absorbance, $I_{T}$ and $I_{0}$ are the intensities of transmitted and incident light, $\varepsilon$ is the molar extinction coefficient, $C$ the concentration of absorbing species and $d$ the distance the light travels through the sample.

Equation (1) can be easily rewritten into the following form:

$$
A=\varepsilon C d
$$


In infrared spectroscopy, it is common practise to describe the wavelength-dependence of the absorbance $A(\lambda)$. From equation (2) it becomes obvious that $A(\lambda)$ is directly related to the wavelength-dependence of the molar extinction coefficients $\varepsilon(\lambda)$

$$
A(\lambda)=\varepsilon(\lambda) C d
$$

Equation (3a) can be alternatively written as

$$
A(\lambda)=\frac{4 \pi d}{\ln (10) \lambda} \kappa(\lambda)
$$

with $\kappa$ being the imaginary part of the refractive index. $\kappa, n_{r}$ and $n$ are linked via the following equation:

$$
n(\lambda)=n_{r}(\lambda)+i \kappa(\lambda)
$$

The symbol $n$ denotes the complex index of refraction. Furthermore, $n_{r}$ is the real part of the refractive index.

Scattering: One of the preconditions that must be fulfilled in order for Beer-Lambert law to be valid is the absence of scattering. For the sake of clarity, we refer in this study only to elastic scattering processes in which the energy of the scattered photons remain unchanged and only the direction is altered. Elastic scattering of light is well understood and the theory of scattering has been established already decades ago. Furthermore, for complex objects an electromagnetic description of light scattering has been extensively formalised. ${ }^{23-27}$ Scattering of electromagnetic radiation by an object, or a system of objects, occurs as a result of spatial heterogeneity of the refractive index, i.e. variations of the complex refractive index $n$ over a spatial dimension $x$. The literature differentiates between three major forms of elastic light scattering. Rayleigh and Mie scattering are known as scattering by particles with dimensions smaller, or of about the same size as the wavelength $\lambda^{27}$ whereas geometric scattering applies to large objects. Mie scattering is of particular importance in this study. It applies to the particular case of light scattering by an absorbing spherical object ${ }^{20,27}$. The Mie theory turned out to be extremely successful to precisely describe cloud or dust light transmission in metrology and aeronomy, but also in many other areas of research such as astronomy and chemistry for modelling the interaction of starlight with interstellar dust, or to estimate the size of particles in colloidal suspensions. ${ }^{19,28-31}$

An important characteristic of scattering is that singly scattered light components usually have other directions than the original direction of incident light (multiple scattered light can have the same direction). For transmission-type measurements it is thus important to realise that only a very small fraction of scattered light reaches an on-axis detector (see Fig. 1b). The intensity of light as measured by such a detector $I_{T}(\lambda)$ can be thus calculated on the basis of the following formula :

$$
I_{T}(\lambda)=I_{0}(\lambda)-I_{S c a t}(\lambda)-I_{A}(\lambda)
$$


where $I_{0}(\lambda)$ corresponds to the intensity of the incident light of the wavelength $\lambda . I_{\text {Scat }}$ is the energy of the scattered light integrated over all directions of space and $I_{A}(\lambda)$ corresponds to the intensity of light that is absorbed by the sample. Equation (5) shows that only in cases where no scattering occurs, i.e. $I_{\text {Scat }}=0$, transmission-type measurements will obey the Beer-Lambert law. In cases where light is scattered, but the scattering efficiency is independent from the wavelength $\lambda$, or nearly independent over relatively narrow spectral regions in the case of Rayleigh scattering, (i.e. $I_{\text {Scat }}(\lambda) \sim I_{0}(\lambda)$ with $I_{\text {Scat }}(\lambda) / I_{0}(\lambda)=k$, with $\left.0 \leq k \leq 1\right)$ one can still observe the band profiles known from IR absorption spectra. In these cases, the experimental absorbance values are scaled by a wavelength-independent factor. In many cases, however, scattering is wavelength-dependent $\left(I_{\text {Scat }}(\lambda) / I_{0}(\lambda) \neq\right.$ const $)$. Under these conditions transmission-type measurements cannot be used anymore for estimating the absorption properties of a sample.

The concept of extinction: In optics the concept of extinction is often used to describe the quantity of light not reaching the detector: ${ }^{19}$

$$
I_{E x t}(\lambda)=I_{A}(\lambda)+I_{S c a t}(\lambda)
$$

In equation (6) $I_{A}(\lambda)$ and $I_{\text {Scat }}(\lambda)$ denote the wavelength dependencies of the intensity of absorbed and of scattered light, respectively with the latter being integrated over all directions. The concept of extinction differs from the concept given by equation (1) in that it includes also other optical processes (scattering) that can lower the intensity of light recorded by an on-axis detector. In electromagnetism the extinction is convenient to calculate because it corresponds to the interference between the incident and forward scattered light $\left(\theta=0^{\circ}, \theta\right.$ is the scattering angle). ${ }^{19}$ Equations (5) and (6) are equivalent because $I_{0}(\lambda)=I_{T}(\lambda)+I_{E x t}(\lambda)$.

Absorption and scattering are not independent from each other: Absorption and scattering are optical processes that are intertwined via the complex index of refraction of the sample under study (see equation (4)). It is well-established that the efficiency of light scattering can be mainly described by the real part of the refractive index $n_{r}(\lambda)$ which is, among others, dependent on the physical properties of the sample (shape, size and number of scatterers). ${ }^{19}$ Contrary to that, absorption is dominated by the imaginary part of the refractive index $\kappa(\lambda)$ which describes the samples' chemistry (Note that the real and imaginary parts of the refractive index are linked via the Kramers-Kronig relation). The complex nature of the refractive index has substantial consequences for spectral analysis in practise. In a typical IR measurement arrangement, for example in a reflection or transmission-type setup with an on-axis detector, it is impossible to distinguish between light attenuation due to absorption or scattering. As in such experimental setups only the extinction, i.e. the sum of both optical effects can be registered, a classical spectroscopic analysis can be made only in cases where scattering is negligible or completely absent. Such a chemical analysis of the spectra, however, will become possible when scattered light components are guided by a suitable optical arrangement onto the detector. Within the context of the present study we suggest a measurement arrangement consisting of an integrating sphere unit and an off-axis IR sensitive detector. In the following we will show that this arrangement will be helpful to correct for spectral contributions from light scattering in FTIR spectral measurements. 


\section{II.2 The Mie scatter size criterion}

In the previous section we have outlined that a chemical analysis of IR spectra is not applicable in cases where scattering cannot be neglected and the scattering efficiency depends on the wavelength $\lambda$ (i.e. $I_{\text {Scat }}(\lambda) / I_{0}(\lambda) \neq$ const. ). As the scattering properties of spherical absorbing particles obviously depend on their physical parameters such as the real part of the refractive index $n_{r, s}$ and the size (radius, $r$ ), we have investigated this dependency with more detail.

For this purpose we propose to use the Mie scattering formalism for spherical particles which gives the full analytical expression for the intensity of light based on Maxwell's theory of electromagnetism. We will use the general equation (see appendix A) to describe shape and position of bands in IR extinction spectra taking into account the complex nature of the refractive index $n$ (see equation (4)). Firstly, it is assumed that scattering results in a shift of the band maximum which holds true in cases of a small scattering efficiency. Secondly, it is proposed that the shift induced by scattering must be larger than $0.1 \mathrm{~cm}^{-1}$ to be detectable. This definition is somewhat arbitrary but appears quite reasonable in the light of a spectral resolution of $4 \mathrm{~cm}^{-1}$ which is commonly used in FTIR spectroscopy of condensed matter with biological samples as a particular case. In appendix B we show that band shifts are not observable (i.e. $\leq 0.1 \mathrm{~cm}^{-1}$ ) when the following condition is satisfied:

$$
m \cdot r \leq \frac{\lambda}{2 \pi}
$$

In this expression $m$ is the ratio between the real part of the refractive index of the sphere $n_{r, s}$ and the corresponding value of the surrounding medium (air) $n_{r, a}$. The radius of the sphere is denoted by $r$ and $\lambda$ is the wavelength of light. At this point it should be emphasised that the Mie scatter size condition was obtained by assuming a band shift not larger than $0.1 \mathrm{~cm}^{-1}$. We have mentioned earlier that $0.1 \mathrm{~cm}^{-1}$ is a rather arbitrary value, but additional simulations have, however, shown that the above-mentioned condition holds true also for smaller band shifts e.g. for $0.01,0.001 \mathrm{~cm}^{-1}$ or even for infinitesimal band shifts. Condition (7) can be thus considered a general criterion that applies to any Mie scattering-associated spectral alteration, including band shifts that are equal or smaller than $0.1 \mathrm{~cm}^{-1}$. Noticeably, the criterion of expression (7) somewhat resembles the wellknown near-field criterion in optics where the evanescent waves dominate the electric field. ${ }^{32}$ It means that the electric field inside a sphere is mainly described by the distribution of evanescent waves in cases where the size of the sphere is small compared to the wavelength. In cases where inequality (7) holds true, the pattern corresponding to the scattered electric field becomes isotropic and nearly independent from the wavelength so that transmission-type measurements allow to obtain interpretable band profiles, i.e. spectra in which the Mie scattering effects are negligible.

The dependence of the critical radius $r$ from the wavelength $\lambda$ has been illustrated in Fig. 2 for a set of spherical absorbing microparticles having different refractive indices $n_{r, s}$. Considering poly(methyl methacrylate) (PMMA) particles of a refractive index of $n=1.3,{ }^{17}$ we can predict that typical PMMA absorbance features such as the $v_{\mathrm{sy}} \mathrm{CH}$ stretching band of $>\mathrm{CH}_{2}$ groups $\left(2850 \mathrm{~cm}^{-1}\right.$, $\lambda=3.51 \mu \mathrm{m})$ will be undisturbed if the radius $r$ of the beads is equal or smaller than $0.43 \mu \mathrm{m}$ (tt- 
FTIR setup, case (a) of Fig. 2). Distortions will be only present in cases where larger spheres are studied. Similarly, in the region of the carbonyl band $\left(1731 \mathrm{~cm}^{-1}, 5.78 \mu \mathrm{m}\right)$ we will find Mie scatter induced spectral alterations only for particles having a radius larger than $0.71 \mu \mathrm{m}$ (case (b) of Fig. 2).

\section{Experimental}

PMMA samples: Monodisperse poly(methyl methacrylate) (PMMA) beads of varying sizes were obtained from microParticles $\mathrm{GmbH}$ (Berlin, Germany). The size of the particles was specified by the manufacturer as follows: (i) $0.242 \pm 0.006 \mu \mathrm{m}$, (ii) $0.404 \pm 0.010 \mu \mathrm{m}$, (iii) $0.615 \pm 0.02 \mu \mathrm{m}$, (iv) $1.04 \pm 0.03 \mu \mathrm{m}$, (v) $2.31 \pm 0.06 \mu \mathrm{m}$, and (vi) $4.86 \pm 0.10 \mu \mathrm{m}$ (average radius \pm standard deviation). The PMMA reference sample has been prepared from a PMMA-chloroform solution. This solution was deposited onto a ZnSe window and allowed to air-dry. The thickness of the PMMA film sample was approximately $2 \mu \mathrm{m}$.

Aqueous suspensions containing the PMMA spheres were diluted in deionised water and deposited for drying on $\mathrm{ZnSe}$ windows. Reducing the original concentration of the microparticles was necessary to exclude contacts between, and stacking of individual PMMA microspheres. Representative light-microscopy photographs of the PMMA microsphere preparations were obtained from the air-dried samples (see Fig. 1S of the supporting information). These photographs demonstrate the absence of stacking and illustrate that contacts between individual microspheres can be considered an exception. The dried samples were characterised in FTIR transmission mode (tt-FTIR) and by the help of an integrating sphere unit (is-FTIR).

FTIR transmission-type measurements (tt-FTIR): These measurements were carried out in transmission mode using a Bruker Vertex 70 FTIR spectrometer (Bruker Optics GmbH, Rheinstetten, Germany), which was equipped with a sensitive liquid nitrogen cooled MCT detector. Spectra were recorded in the spectral range between 400 to $4000 \mathrm{~cm}^{-1}$ ( $\left.\lambda=25-2.5 \mu \mathrm{m}\right)$. The nominal spectral resolution used was $4 \mathrm{~cm}^{-1}$. Altogether 100 scans were co-added per sample spectrum and were apodised using a Happ-Genzel apodisation function for Fourier transformation. Interferograms were zero-filled using a zero filling factor of 4 to yield an encoding interval of approximately one data point per wavenumber. Spectra of the tt-FTIR measurements were vector-normalized (2-norm) in order to compensate for the unavoidable variations of the concentration of the microparticles.

FTIR measurements by an integrating sphere unit (is-FTIR): is-FTIR measurements have been realised by means of an integrating sphere unit from Bruker (A 562-G/Q, Bruker Optics GmbH, Rheinstetten, Germany). The unit was originally designed for diffuse reflection measurements and was coated with a layer of diffuse reflecting gold. The IR signal was recorded by the help of a midinfrared off-axis DLaTGS (deuterated L-alanin doped triglycine sulfate) detector with an element size of $2.5 \times 2.5 \mathrm{~mm}^{2}$ operating at room temperature. Parameters of spectral data acquisition (resolution, scan numbers, spectral range, apodisation function, etc.) were the same as for the ttFTIR measurements. Again, spectra were vector-normalized for reason of comparison.

Numerical simulations: The calculation of the scattering efficiency $Q_{\text {ext }}$ and of the apparent absorbance has been performed by a program written in $\mathrm{C}$ developed in-house by one of us (A.D.). 
In order to obtain good convergence, spherical Bessel and Hankel functions were developed by a series expansion until the $10^{\text {th }}$ order term. Another $\mathrm{C}$ code program has been written by A.D to determine the values of the radii $r$ for a series of spheres with variable refractive indices $n_{s}$. To simulate the peak shift of a synthetic absorption band as a result of scattering, an iterative procedure was employed in which combinations of $n_{s}$ and $r$ values were systematically varied until the superposition of absorptive and scattering line shapes resulted in a peak shift of $0.1 \mathrm{~cm}^{-1}$. The peak shift was obtained as the difference between the positions of the maxima of the mixed and the pure absorptive reference band.

\section{Results}

One of the main objectives of the present study was to systematically examine the absorption and scattering properties of microparticles of known chemical composition in the mid-infrared spectral range. In this context the dependence of the scattering properties on the size (radius, $r$ ) of the microparticles and the analysis of the question whether the disturbing influence of Mie scattering can be suppressed by a suitable measurement arrangement were of particular interest. To answer these questions monodisperse poly(methyl methacrylate) (PMMA) beads of various radii $(0.242 \geq r \geq 4.86 \mu \mathrm{m})$ were characterised by means of the classical transmission-type FTIR measurement technique (tt-FTIR) and alternatively, by the help of an integrating sphere unit (isFTIR). While in the case of tt-FTIR light is collected only in the direction of incoming (incident) light, the is-FTIR technique allowed to collect the signal from transmitted and forward-scattered light components (cf. Fig. 1b and 1c).

Fig. 3 shows a reference spectrum of a thin and homogeneous PMMA polymer film prepared on a $\mathrm{ZnSe}$ substrate. This spectrum was recorded in IR transmission mode and exhibits on a flat spectral baseline a number of intense IR absorption bands. Vibrational bands of this spectrum were found in good agreement with the literature values. ${ }^{33-35}$ Infrared bands arising from methylene and methyl groups $\left(v(\mathrm{CH})\right.$ of $>\mathrm{CH}_{2}$ and $\left.-\mathrm{CH}_{3}\right)$ were observed at short wavelengths between 2800 and $3000 \mathrm{~cm}^{-}$ ${ }^{1},(3.57-3.28 \mu \mathrm{m})$ whereas the intense carbonyl band was found at $1731 \mathrm{~cm}^{-1}(5.78 \mu \mathrm{m})$. At longer wavelengths $\left(1000-1400 \mathrm{~cm}^{-1}, 10-7.14 \mu \mathrm{m}\right)$ one can detect a number of vibrational modes from C-C-O or C-O-C groups (see Tab. 1 for details of band assignments in this region).

FTIR spectra from the transmission-type (tt-FTIR) and the integrating sphere (is-FTIR) measurements are shown in Fig. 4. These spectra have been obtained from diluted PMMA bead preparations which were mounted by air-drying on a ZnSe substrate (cf. Fig 1S). In Fig. 4 spectra were shifted along the y-axis for clarity. Traces 1 of Fig. 4 demonstrate IR spectra from PMMA beads of a radius of $0.242 \mu \mathrm{m}$. Both IR spectra exhibit a relatively high level of similarity with the PMMA reference spectrum of Fig. 3. The latter finding indicates only little influence of light scattering on the characteristics of both, the tt-FTIR and is-FTIR spectrum. It must be noted however, that the SNR of the is-FTIR spectrum is relatively low, possibly a result of a low concentration of PMMA beads and the generally reduced intensity in the is-FTIR measurement configuration. The tt-FTIR spectrum and the is-FTIR spectrum of PMMA microparticles of a radius of $0.404 \mu \mathrm{m}$ are given by traces 2 of Fig. 4 . As in the previous case, vibrational bands of PMMA 
are found within the accuracy of the measurements at their exact wavenumber positions. At short wavelengths $\left(>3000 \mathrm{~cm}^{-1}\right.$, or $\left.<3.33 \mu \mathrm{m}\right)$ the tt-FTIR spectrum shows a noticeable baseline slope. Traces 3 of Fig. 4 display the tt-FTIR and the is-FTIR spectrum recorded from PMMA spheres with a radius of $0.615 \mu \mathrm{m}$. Spectra are still remarkably similar but the baseline slope of the tt-FTIR spectrum is more pronounced and can be observed also at longer wavelengths $\left(\sim 1800 \mathrm{~cm}^{-1}, 5.56\right.$ $\mu \mathrm{m})$. Contrary to that, the is-FTIR measurement resulted in a spectrum with a flat baseline and nearly unchanged spectral bands. Spectra of monodisperse PMMA beads with a radius of $1.04 \mu \mathrm{m}$ are given by traces 4 of Fig. 4. The tt-FTIR spectrum exhibits a markedly distorted baseline at wavelengths below $10 \mu \mathrm{m}$. Furthermore, the shape of the carbonyl band has become asymmetric with a negative side lobe at the short wavelength side. These spectral changes are in good agreement with earlier observations of Bassan et al. ${ }^{17}$, who found similar changes in IR microspectra of individual PMMA microspheres. Contrary to the findings, the is-FTIR spectrum showed no significant differences compared to the pure absorption spectrum of Fig. 3. FTIR spectra obtained from PMMA beads of a radius of $2.31 \mu \mathrm{m}$ are given by traces 5 of Fig. 4 . As it can be taken from the stronger baseline slope and inverted band features in the $\mathrm{CH}$-stretching region, the ttFTIR spectrum is strongly influenced by scattering. Furthermore, scattering-induced alterations in the carbonyl band region are so intense that the carbonyl band resembles a derivative-like line shape rather than a Lorentzian band profile. The band shift, by measuring the position at the band maximum, reaches a value of $-11 \mathrm{~cm}^{-1}\left(1720 \mathrm{~cm}^{-1}\right.$ compared with $1731 \mathrm{~cm}^{-1}$ when scattering is absent). In trace 5 (left panel) spectral alterations are not anymore limited to the $\mathrm{CH}$ stretching region, or the carbonyl band: other bands at longer wavelengths $\left(>6 \mathrm{~cm}^{-1}\right)$ are also affected, albeit to a lesser extent. The C-C-O and C-O-C vibrations (see Tab. 1) are markedly degraded as seen by apparent changes in intensity, position and number of peaks. Measurements of beads of a radius of $2.31 \mu \mathrm{m}$ in the is-FTIR configuration resulted again in a spectrum with a flat baseline and bands with only little modified spectral characteristics (see trace 5 of Fig. 4, right panel).

Traces 6 of Fig. 4 depict tt-FTIR and is-FTIR spectra for spheres with a radius of $4.86 \mu \mathrm{m}$. The isFTIR spectrum shows again a flat baseline and vibrational bands at their correct positions. It should be mentioned, however, that some band broadening along with alterations of the relative intensity ratios are observable (which could be a result of saturation effects, possibly because of large optical path lengths for individual microparticles, $d=2 r$ ). In contrast to this the changes in the tt-FTIR spectrum are indeed remarkable: instead of a single carbonyl band at $1731 \mathrm{~cm}^{-1}$ one can observe an inverted band doublet. Furthermore, IR bands at longer IR wavelengths ( $>6 \mu \mathrm{m})$ exhibit reduced relative intensities, peak shifts and derivative-like line shapes indicating strong scattering effects also in this spectral region. Interestingly, at shorter wavelengths $(<5 \mu \mathrm{m})$ one can now see a decrease of scattering-associated features. Such alterations are surprisingly moderate in the C-H stretching region of the tt-FTIR spectrum. Negative peaks of the $\mathrm{C}-\mathrm{H}$ stretching region (cf. trace 5) became positive again and band positions can be found close to the expected values. 


\section{Discussion}

Scattering-associated spectral changes in the mid-infrared region have been described by many authors in a number of studies. ${ }^{7,8,15-18}$ Typical spectral characteristics of Mie scattering are broad baseline effects (oscillating baselines, baseline slopes) and sharp scattering features (e.g. derivativelike line shapes, band shifts, inverted bands, etc.). These spectral changes are often dramatic and can significantly complicate the analysis and interpretation of the data.

A variety of different terms such as "dispersion artefact", "anomalous dispersion", or "(Resonant) Mie scattering" can be found in the literature to describe the wealth of scattering-induced spectral alterations. We and others ${ }^{22}$ believe that "Resonant Mie scattering" should preferably be used to describe such effects because this term includes both, the presence of Mie scattering and the fact that the real and the imaginary part vary together in "resonance" in the region of an absorption band. Other terms ("dispersion artefact", "anomalous dispersion" ${ }^{7,17}$ ) should be avoided in the future because they more likely confuse than clarify the complex subject.

Besides the potential application of an integrating sphere for reducing light scattering contributions (see below) it was also important to us to understand the relationships between the wavelengthdependent spectral changes of the PMMA microspheres and physical parameters, the radius $r$ and the refractive index $n$, of our model objects. In this regard it was possible to deduce a theoretical relationship, the Mie scatter size criterion, from simulated data and to experimentally confirm the validity of this dependency. It was found that the Mie scatter size criterion represents a threshold of the size of spherical microparticles that describes the occurrence of Mie scattering phenomena in a way similar to the well-known near field criterion. While light scattering by microparticles larger than the Mie scatter size criterion can be explained by the Mie theory, scattering by smaller spheres follows the laws of Rayleigh scattering.

We now turn to a brief discussion of the experimental results and a comparison of the classical ttFTIR and the is-FTIR measurement technique. The experimental data acquired using the tt-FTIR data acquisition setup from the chemically uniform, but physically heterogeneous PMMA microspheres demonstrate the large spectral changes caused by the optical process of scattering. As shown by traces 1-6 of Fig. 4 (tt-FTIR setup, left panel) variations of the size of the PMMA microspheres resulted in enormous spectral effects: baseline slopes including oscillating/undulating baselines, peaks shifts, derivative-like line shapes, band splitting and even inverted IR bands can significantly modify the characteristic band profiles of tt-FTIR spectra and considerably complicate the interpretation of the spectra in terms of biochemical structure and composition. These observations are not new to the field of biomedical IR spectroscopy. PMMA microspheres have been used by others as excellent surrogates to model scattering from cells and cell nuclei. ${ }^{17}$ Furthermore, scattering is known since a long time in the field of biomedical IR spectroscopy and has been thus a subject of intense research. ${ }^{7,15,17-18}$ In the past, there have been multiple attempts to understand the nature of the spectral changes associated with scattering and to develop physical models to separate absorption and scattering effects in the IR spectra. ${ }^{6-8,15,19}$ The models developed in these studies have been incorporated into computer algorithms to minimise scattering effects in contaminated IR absorption spectra. For example, Bassan et al. applied the van de Hulst 
approximation ${ }^{8}$ and in a subsequent study an improved model with the full Mie scattering formalism for separating scattering and pure absorption contributions from experimental IR spectra ${ }^{22}$. Although these efforts have proved to be exceptionally useful for many practical applications, it cannot be overlooked that the scattering correction routines are rather complex and often not accessible to the practitioners. Furthermore, with calculation times of hours even for small data sets the procedures are computationally demanding and thus only of limited usefulness for large and very large data sets (i.e. for hyperspectral imaging applications). It should be also noted that although the proposed computational methods of scatter correction often improve shape and contour of the experimental spectra, the spectral fine structure, e.g. of the amide I band, may resemble details of the reference spectrum which is required for the correction (observation of the authors when the van de Hulst approximation method was used ${ }^{8,23}$ ).

With this study, we propose to minimise scattering effects in a radically different way. Instead of modelling absorption and scattering properties of the biomedical samples we propose a measurement setup that allows collecting scattered and transmitted light components simultaneously by an integrating sphere unit as the main component of the optical setup. The experimental comparison between the classical tt-FTIR measurement and the is-FTIR measurement on the same scattering sample has successfully proved that contributions from scattering can be indeed removed from the measured spectra. The use of an integrating sphere is not a common approach in the field of mid-infrared spectroscopy, whereas such equipment has been widely applied to measure UV, visible and near-infrared spectra of small particles and powders. ${ }^{36,37}$ The experimental data acquired from the model objects of PMMA microspheres presented in this study demonstrate quite clearly that integrating spheres are suitable measuring devices to minimise scattering contribution: spectra of the right panel of Fig. 4 (is-FTIR) exhibit a high level of similarity with the reference spectrum obtained from the PMMA film sample (see Fig. 3). All of the is-FTIR spectra exhibited pure IR absorptions features on a flat baseline in which the typical broad Mie-type baseline slope, or the narrow RMieS characteristics are clearly absent. Despite these encouraging findings, it shall not be concealed that a number of factors exist which could potentially preclude the use of the is-FTIR data acquisition technology. In this context it is primarily noteworthy, that is-FTIR spectra of Fig. 4 show a considerably reduced signal to noise ratio (SNR) compared with the spectra acquired by means of tt-FTIR (cf. is-FTIR spectra 1 and 2 of Fig. 4). For a discussion of this observation, several factors must be considered. First, it should be noted that the preparation of a suitable sample of PMMA microspheres is not an easy task. To avoid contacts or even stacking of the microparticles, only solutions of a low particle concentration could be handled. This resulted in a limited amount of material being measured in the given experimental setup. We found this consideration particularly relevant for small microparticles, i.e. PMMA spheres of radii of 0.242 and $0.404 \mu \mathrm{m}$. (cf. traces 1 and 2). A second important factor in the assessment of the reduced SNR is that the is-FTIR system is by no means optimised for measuring spectra from microparticles: a large amount of IR intensity is lost due to multiple (diffuse) reflections at the inner surface of the sphere. Thirdly, the different sensitivities of the detectors used for tt-FTIR and isFTIR should be also taken into account. While in the tt-FTIR measurements, a MCT detector was employed, the integrating sphere unit was equipped with a large-area DLaTGS detector. Although 
we do not find ourselves in the position to assess whether the higher sensitivity of the MCT detector compensates for the larger size of the DLaTGS detector $(2.5 \times 2.5 \mathrm{~mm}$ !) it is however, beyond any doubt that the amount of light that reaches the detector could be more sensitively registered when the integrating sphere unit would be equipped with one or more large area MCT detectors.

For a practical application of is-FTIR instrumentation, for example in IR microscopy and IR imaging, it is in our view absolutely necessary to implement technical measures to achieve a higher sensitivity. Besides the aforementioned way to improve IR detection this could be achieved via alternative coatings of improved reflectivity, more intense IR sources (synchrotron), or adapted optical solutions, for examples by utilising optimised geometries to collect and transmit more of the isotropic radiation onto the detector.

In case of success, both, computer-based ways and instrumental options would be available to separate absorption and scattering contributions in the measured mid-infrared spectra.

\section{Conclusions}

In this paper, we have demonstrated that distorted mid-infrared spectral line shapes arising from scattering can be minimised by using an optical system consisting of an integrating sphere unit, an off-axis DLaTGS detector and a commercial Fourier transform infrared (FTIR) spectrometer. This system turned out to be an attractive alternative to computer-based methods of scatter correction if the sample under study can be conveniently deposited on the optical substrate. The main advantage of the method is that absorbance spectra can be directly recorded, which contain only compositional and structural information (absorbance) from which the physical (scattering) information has been eliminated. This obviates the need for using complex and time-consuming computer-based correction methods. For the future it is intended to further develop the proposed methodology and to incorporate an integrating sphere unit into a standard FTIR spectrometer. This should allow for simple sample preparation and collection of high-quality absorbance spectra from strong scatterers also in a microspectroscopic setup. 


\section{Supplementary material}

\section{APPENDIX A}

The first attempts to establish a theory of absorption and scattering for spherical objects go back to 1908 when Gustav Mie tried to explain colour variations exhibited by small gold particles suspended in water. ${ }^{19,20}$ Since then, a number of authors have worked on this topic and provided a modern formulation of the Mie-theory (see refs. ${ }^{26,38}$ for example). In this appendix we will present the classical mathematical approach that can express both, absorption and scattering of light by a sphere of a radius $r$ with a complex index of refraction $n_{s}$.

The extinction efficiency $Q_{\text {ext }}$ of a spherical object of radius $r$ with a complex refractive index $n_{s}$ surrounded by a medium of a complex refractive index $n_{a}$ corresponds to the extinction cross section per unit area. The extinction efficiency can be written in the following way: ${ }^{19}$

$$
Q_{\text {ext }}=\frac{2}{x^{2}} \sum_{i=1}^{\infty}(2 i+1) \Re\left(a_{i}+b_{i}\right)
$$

with

$$
x=\frac{2 \pi}{\lambda} n_{a} r
$$

In equation (A.1) $a_{i}$ and $b_{i}$ denote the scattering coefficients of the electric field. The expressions of the scattering coefficients are a combination of Riccati-Bessel functions. ${ }^{19}$

$$
\begin{aligned}
& a_{i}=\frac{m^{2} x j_{i}(m x)\left(x j_{i}(x)\right)^{\prime}-x j_{i}(x)\left(m x j_{i}(m x)\right)^{\prime}}{m^{2} x j_{i}(m x)\left(x h_{i}(x)\right)^{\prime}-x h_{i}(x)\left(m x j_{i}(m x)\right)^{\prime}} \\
& b_{i}=\frac{m x j_{i}(m x)\left(x j_{i}(x)\right)^{\prime}-m x j_{i}(x)\left(m x j_{i}(m x)\right)^{\prime}}{m x j_{i}(m x)\left(x h_{i}(x)\right)^{\prime}-m x h_{i}(x)\left(m x j_{i}(m x)\right)^{\prime}}
\end{aligned}
$$

where $m=n_{s} / n_{a}, j_{i}$ is the spherical Bessel function and $h_{i}$ is the spherical Hankel function. Using this formalism it is possible to obtain the precise wavelength-dependence of the extinction of a sphere. Conversion between extinction and absorbance was carried out by using the following relationship:

$$
A=-\log _{10}\left(1-Q_{e x t}\right)
$$

In equation (A.3) $A$ denotes the so-called "apparent absorbance", a term suggested by Bassan et al. ${ }^{17}$ which involves both, attenuation due to absorption and scattering.

Application of the formalism to an absorbing sphere with one absorption band: In the following example we assume spheres of various sizes having a refractive index $n_{s}$ of $1.5\left(\mathrm{n}_{\mathrm{s}}=\mathrm{n}_{\mathrm{r}, \mathrm{s}}+\mathrm{i} \kappa_{\mathrm{s}}\right)$. We assume furthermore absorption of light by the spheres giving rise to a Lorentzian-shaped absorption band which is centred at $\lambda=5 \mu \mathrm{m}\left(2000 \mathrm{~cm}^{-1}\right)$ and is characterised by a full width at half maximum of $0.01 \mu \mathrm{m}\left(20 \mathrm{~cm}^{-1}\right)$. We consider also the wavelength dependence of both, the imaginary and real parts of the refractive index $\left(n_{r, s}(\lambda), \kappa_{s}(\lambda)\right.$, see Fig. A). 
The calculation of the extinction efficiency $Q_{\text {ext }}$ as a function of the wavelength according to equation A.1 and the apparent absorbance (see eqn. (A.3)) will directly give us the theoretical spectrum of the sphere recorded in a classical infrared transmission type setup, i.e. tt-FTIR with onaxis detection. It should be emphasised that the solutions obtained by this approach represent the full analytical expressions of the Mie theory, i.e. no approximations have been utilised for calculating the scattering coefficients.

Fig. B shows the simulated apparent absorption spectra for spheres of various radii in the region between 1800 and $2200 \mathrm{~cm}^{-1}(5.56-4.55 \mu \mathrm{m})$. The lower trace of Fig. B represents the reference spectrum (no scattering) with the synthetic absorption band centred at $2000 \mu \mathrm{m}$. The next spectrum has been calculated for a microsphere of a radius of $0.5 \mu \mathrm{m}$. The size of this particle is close to the theoretical Mie scatter size criterion (equals $0.53 \mu \mathrm{m}$, vide infra) so that scattering-induced spectral alterations are barely detectable in this case. The same statement holds true also for the microparticle with a radius of $0.6 \mu \mathrm{m}$ (see Fig. B). As the particle size further increases $(\mathrm{r}=0.8 \mu \mathrm{m})$ we can observe a gradual introduction of scattering features. As seen by Fig. B such features initially involve some band asymmetry associated with a minor band shift. With increasing particle size, the alterations get larger until a derivative-like line shape can be observed for a particle with a radius of $2.0 \mu \mathrm{m}$. It is quite obvious that such tremendous spectral changes cannot be analysed and interpreted in the classical way.

The effect of scattering is well-described for spherical objects, but there are many other mathematical treatments available which can be used to describe scattering on objects with cylindrical, oblate-like, or some other symmetrical shape. Similarly to spherical objects and regardless of the shape of the scatterers, scattered light will have a wavelength distribution which does not necessarily correspond to the original distribution of the incident light. Degradation of spectral absorption features is therefore not limited to scattering by spherical objects to which the Mie theory is restricted. For scatterers of various sizes with irregular shape - such as biological objects - it is therefore practically not possible to establish solutions to computationally separate between contributions from absorption and scattering.

With the subsequent second section (appendix B) we provide an example of how the full Mie approach of light scattering by spherical particles can be utilised to determine the critical particle size at which the above-mentioned degradation of spectral features will be absent.

\section{APPENDIX B}

In appendix $\mathrm{A}$ it was demonstrated that the amplitude of spectral degradation due to scattering depends on the size of the spherical scatterers. The main goal of this section was thus to investigate the question, whether thresholds exist at which no Mie scattering-associated spectral alterations are detectable.

To this aim a computer program has been developed which was used to model the band shift arising from mixing of real (scattering) and imaginary (absorption) band shapes. Again, we have assumed spheres of various sizes (radius $r$ ) having a complex refractive index $n_{s}$. It was also assumed that the microspheres are composed of a material giving an IR band centred at $\lambda=5 \mu \mathrm{m}\left(2000 \mathrm{~cm}^{-1}\right)$ with the band having a full width at half height of $0.01 \mu \mathrm{m}\left(20 \mathrm{~cm}^{-1}\right)$. The microspheres are illuminated 
by collimated light in the wavelength range between 1800 and $2200 \mathrm{~cm}^{-1}(5.56-4.55 \mu \mathrm{m})$. For the sake of simplicity it was defined that only peak shifts larger than $0.1 \mathrm{~cm}^{-1}$ can be detected at our experimental conditions.

Using the framework given by appendix A we have then calculated the thresholds of microsphere radii $r$ for a given set of refractive indices $n_{r, s}$ ranging from $1.1-1.7$ considering that the surrounding medium is air $\left(n_{r, a}=1\right)$. The thresholds of $r$ for the different refractive indices are given in Tab. 2. A closer inspection of the dependence $r$ from $n_{r, s}$ clearly revealed a linear dependency with an average product $\left\langle n_{r, s} r\right\rangle$ of 0.786 with a standard deviation of 0.03 . If we then introduce the wavelength dependence of $r$ to the simulation, we can finally obtain a condition that links $n_{r, s}, r$ and $\lambda$ :

$$
n_{r, s} r<0.157 \cdot \lambda
$$

In equation B. 1 the value 0.157 was obtained by dividing 0.786 by the wavelength $\lambda\left(2000 \mathrm{~cm}^{-1}\right.$ or $5 \mu \mathrm{m})$. Although expression B.1 has been obtained to estimate if Mie scattering-associated degradation can be observed in the IR spectra, the condition is also valid for other wavelength regions, including the wavelength region of visible light.

The value of 0.157 is not a random value and it can be shown that this parameter is linked to a wellknown optical parameter. In analogy to near-field optics where the near-field region is defined by a distance that is smaller than the wavelength $\lambda$ divided by $2 \pi$, one could discuss the value of 0.157 also as $1 / 2 \pi$. In conclusion, we propose that the criterion given in equation B. 1 is linked with the well-known near-field definition and can be defined in analogy to ${ }^{32}$ by the following equation:

$$
m \cdot r \leq \frac{\lambda}{2 \pi}
$$

In this equation $m$ is the ratio between $n_{r, s}$ and $n_{r, a}$, which is defined as the real part of refractive index of the surrounding medium (air $n_{r, a}=1$ ). The analogy between the definition of the near-field region and the Mie scatter size criterion of equation B.2 is quite obvious. The Mie scatter size criterion is linked with the near field criterion by the fact that scattering by microparticles always involves forward and backward propagatives waves inside the sphere. These waves can interfere with the incident wave. If the size of the scattering particles is smaller than the Mie scatter size criterion of equation B.2 then interference and consequently Mie scattering cannot be observed anymore. In such cases scattering from small-sized spherical particles is explained by the Rayleigh theory. 


\section{Legends to the Figures}

\section{Figure 1}

A. Classical transmission-type FTIR measurement setup (tt-FTIR) of a homogeneous sample of a thickness $d$ in the absence of scattering. Transmitted light is recorded by an on-axis IR detector.

B. Same tt-FTIR measurement setup on a sample showing spatial heterogeneity of its refractive index $n$. Scattered light components do not contribute to the detector signal.

C. FTIR measurement of heterogeneous samples using an integration sphere whose interior is coated with a layer of diffusing, non-absorbing gold and a sensitive off-axis detector (is-FTIR). The gold layer reflects incident light from transmitted and forward-scattered light components into all possible directions, resulting in homogeneous and isotropic radiation inside the sphere.

\section{Figure 2}

The Mie scatter size criterion for a set of spherical microparticles having various refractive indices $n_{r, s}$ illuminated by light of a wavelength $\lambda$. The criterion defines a critical radius $r$ above which Mie scattering becomes significant. For simplicity, the refractive index of the surrounding medium $n_{r, a}$, was set to 1 (air, see also text for details).

\section{Figure 3}

Typical FTIR absorbance spectrum collected from a chemically homogeneous film of poly(methyl methacrylate) (PMMA, see Tab. 1 for band assignments).

\section{Figure 4}

Vector normalized mid-IR spectra acquired from poly(methyl methacrylate) (PMMA) spheres using a FTIR transmission-type measurement setup (tt-FTIR, left panel) and an optical system composed of an integrating sphere unit equipped with an off-axis IR detector (is-FTIR, right panel). Only the radii of the PMMA spheres were varied. Note that in this figure the y-axis is labelled "apparent absorbance" which includes both, absorption and scattering effects ${ }^{17}$. Radii of the PMMA spheres: trace $1-0.242 \mu \mathrm{m}$, trace $2-0.404 \mu \mathrm{m}$, trace $3-0.62 \mu \mathrm{m}$, trace $4-1.04 \mu \mathrm{m}$, trace $5-2.31 \mu \mathrm{m}$, trace $6-4.86 \mu \mathrm{m}$.

\section{Figure A}

Simulated behaviour of the wavelength dependence of the real part of the refractive index $\left(n_{r, s}\right.$, trace i) and of the imaginary part $\left(\kappa_{\mathrm{s}}\right.$, trace ii). The simulation was done assuming a Lorentzian band centred at $\lambda=5 \mu \mathrm{m}\left(2000 \mathrm{~cm}^{-1}\right)$ with a full width at half maximum of $0.01 \mu \mathrm{m}\left(20 \mathrm{~cm}^{-1}\right.$, see appendix A for details).

\section{Figure B}

Simulated spectra (tt-FTIR setup) demonstrating band distortions due to absorption and scattering on spheres of various radii. In this synthetic example the wavelength dependent apparent absorption 
curves clearly show a nearly unchanged spectral profile at radii smaller than $\lambda /(2 \pi n)$. For spheres of a larger radius the apparent absorption curves are dominated by scattering. 


\section{Tables}

Table 1

\begin{tabular}{|c|c|c|}
\hline Band position $\left[\mathrm{cm}^{-1}\right]$ & Band assignment & References \\
\hline 2997 & $v(\mathrm{CH})$ of $\mathrm{O}-\mathrm{CH}_{3}$ and $\mathrm{C}-\mathrm{CH}_{3}$ & $17,34,35$ \\
\hline 2950 & $v(\mathrm{CH})$ of $\mathrm{CH}_{2}, \mathrm{O}-\mathrm{CH}_{3}$ and $\mathrm{C}-\mathrm{CH}_{3}$ & $17,34,35$ \\
\hline 2851 & $v(\mathrm{CH})$, of $\mathrm{CH}_{2}$ and $\mathrm{O}-\mathrm{CH}_{3}$ & $17,34,35$ \\
\hline 1731 & $v(\mathrm{C}=\mathrm{O})$ & 17,34 \\
\hline 1482 & $\delta\left(\mathrm{CH}_{2}\right)$ (scissoring) & 17,35 \\
\hline 1450 & $\begin{array}{l}\delta\left(\mathrm{CH}_{2}\right) \text { (scissoring), } \delta_{\text {as }}\left(\mathrm{CH}_{3}\right) \text { of } \mathrm{O}-\mathrm{CH}_{3} \text { and } \\
\mathrm{C}-\mathrm{CH}_{3}\end{array}$ & 17,35 \\
\hline 1442 & $\delta_{\text {sy }}\left(\mathrm{CH}_{3}\right)$ of O- $\mathrm{CH}_{3}$ (umbrella) & 17,35 \\
\hline 1388 & $\delta_{\text {sy }}\left(\mathrm{CH}_{3}\right)$ of C-CH (umbrella) & 17,35 \\
\hline 1271,1241 & $\mathrm{v}_{\mathrm{as}}(\mathrm{C}-\mathrm{C}-\mathrm{O})$ & 17 \\
\hline 1191,1150 & $v_{\text {as }}(\mathrm{C}-\mathrm{O}-\mathrm{C})$ & 17 \\
\hline 1065 & skeletal $v(\mathrm{C}-\mathrm{C})$ & 34 \\
\hline 987 & $\mathrm{O}-\mathrm{CH}_{3}$ rocking coupled with $v(\mathrm{C}-\mathrm{O}-\mathrm{C})$ & 34 \\
\hline 844 & $\mathrm{CH}_{2}$ rocking & 34 \\
\hline 755 & skeletal $v(\mathrm{C}-\mathrm{C})$ & 34 \\
\hline
\end{tabular}

Table 1. Assignment of infrared bands observed in an absorbance spectrum of a poly(methyl methacrylate) reference sample, a thin PMMA film. 
Table 2

\begin{tabular}{|c|c|l|}
\hline$n_{s}$ & $r[\mu \mathrm{m}]$ & $n_{r, s}{ }^{*}[\mu \mathrm{m}]$ \\
\hline 1.10 & 0.68000 & 0.74800 \\
\hline 1.15 & 0.65000 & 0.74750 \\
\hline 1.20 & 0.63000 & 0.75600 \\
\hline 1.25 & 0.61000 & 0.76250 \\
\hline 1.30 & 0.59000 & 0.76700 \\
\hline 1.35 & 0.58000 & 0.78300 \\
\hline 1.40 & 0.56000 & 0.78400 \\
\hline 1.45 & 0.55000 & 0.79750 \\
\hline 1.50 & 0.54000 & 0.81000 \\
\hline 1.55 & 0.52000 & 0.80600 \\
\hline 1.60 & 0.51000 & 0.81600 \\
\hline 1.65 & 0.50000 & 0.82500 \\
\hline 1.70 & 0.48000 & 0.81600 \\
\hline
\end{tabular}

Table 2. Critical values of microsphere radii $r$ for a given set of refractive indices $n_{r, s}$. Simulations were carried out using the full Mie scattering theory and under the premise that mixing of absorptive and scattering line shapes results in a peak shift of a synthetic band centred at $2000 \mathrm{~cm}^{-1}$ $(5 \mu \mathrm{m})$ that is smaller than $0.1 \mathrm{~cm}^{-1}$ (see text for details). 


\section{References}

[1.] Detectors for Fourier Transform Spectroscopy. Thermo Scientific Application Note 50808. 2007.

[2.] J. M. Kwiatkoski and J. A. Reffner, Nature, 1987, 328, 837 - 838.

[3.] J.A. Reffner, P.A. Martoglio and G.P. Williams, Rev. Sci. Instrum., 1995, 66, 1298.

[4.] E.N. Lewis, P.J. Treado, R.C. Reeder, G.M. Story, A.E. Dowrey, C. Marcott and I.W. Levin. Anal Chem., 1995, 67, 3377-81.

[5.] H. Martens and E. Stark E, J Pharm Biomed Anal., 1991, 9, 625-35.

[6.] H. Martens, J.P. Nielsen and S.B. Engelsen, Anal. Chem., 2003, 75, 394-404.

[7.] M. Romeo and M. Diem, Vib. Spectrosc., 2005, 38, 129-132.

[8.] Bassan P, Kohler A, Martens H, Lee J, Byrne HJ, Dumas P, Gazi E, Brown M, Clarke N and Gardner P. Analyst, 2010, 135, 268-277.

[9.] P. Lasch, Chemom Intell Lab Syst., 2012, 117, 100-114.

[10.] D. Helm, H. Labischinski, G. Schallehn and D. Naumann, J Gen Microbiol., 1991, 137, 6979.

[11.] D. Naumann, D. Helm and H. Labischinski, Nature, 1991, 351; 81-2.

[12.] P. Lasch, M. Diem, W. Hänsch and D. Naumann, J. Chemometrics, 2006, 20; 209-220.

[13.] P. Lasch, W. Haensch, D. Naumann and M. Diem, Biochim Biophys Acta., 2004, 1688, 17686.

[14.] T. Chernenko, C. Matthäus, L. Milane, L. Quintero, M. Amiji and M. Diem, ACS Nano, 2009, 3, 3552-9.

[15.] M. Miljković, B. Bird and M. Diem, Analyst, 2012, 137, 3954-64.

[16.] B. Mohlenhoff, M. Romeo, M. Diem and B.R. Wood Biophys J., 205, 88, 3635-40.

[17.] P. Bassan, H.J. Byrne, F. Bonnier, J. Lee, P. Dumas and P. Gardner, Analyst, 2009, 134, 1586-1593.

[18.] J. Lee, E. Gazi, J. Dwyer, M.D. Brown, N.W. Clarke, J.M. Nicholson and P. Gardner, Analyst, 2007, 132, 750-755.

[19.] C.F. Bohren and D.R. Huffman, Absorption and Scattering of Light by Small Particles, Wiley-VCH, Weinheim/Germany, 1983.

[20.] G. Mie, Annalen der Physik, 1908, 330, 377.

[21.] A. Kohler, J. Sulé-Suso, GD. Sockalingum, M. Tobin, F. Bahrami, Y. Yang, J. Pijanka, P. Dumas, M. Cotte, DG. van Pittius, G. Parkes and H. Martens, Appl Spectrosc., 2008, 62, 25966.

[22.] P. Bassan, A. Kohler, H. Martens, J. Lee, E. Jackson, N. Lockyer, P. Dumas, M. Brown, N. Clarke and P. Gardner, J Biophotonics, 2010, 3, 609-20.

[23.] H. C. Van De Hulst, Light Scattering by Small Particles, Dover Publications Inc. Mineola, NY, 1981.

[24.] M. Neviere and E. Popov, Optical Science and Engineering, Marcel Dekker Inc. Ed., 2002.

[25.] L. Novotny and B. Hecht, Principles of Nano-Optics, Cambridge University Press, 2006.

[26.] M. Kerker, The Scattering of Light and Other Electromagnetic Radiation, Academic Press, New York, 1969.

[27.] A.B. Pluchino, S.S. Goldberg, J.N. Dowling and C.M. Randall, Appl. Opt., 1980, 19, 33703372.

[28.] P.J. Wyatt, Appl. Opt., 1980, 19, 493-501.

[29.] J.E. Hansen, W.C. Wang and A.A. Lacis, Climatic effects of atmospheric aerosols, In: Proc. Conf. on Aerosols: Urban and Rural Characteristics, Source and Transport studies, New York Academy of Science, NY, 1979.

[30.] N.C. Wickramasinghe and K. Nandy, Rep. Prog. Phys., 1972, 35, 157-234.

[31.] I. Giaver, J. Immunol., 1973, 110, 1424-1426.

[32.] J.J. Greffet and R. Carminati, Prog. Surf. Science, 1997, 56, 133-237.

[33.] H. A. Willis, V. J. I. Zichy and P. Hendra, J. Polymer., 1969, 7, 737.

[34.] S. Dirlikov and J. L. Koenig, Appl. Spectrosc., 1979, 33, 551-555.

[35.] S. Dirlikov and J. L. Koenig, Appl. Spectrosc., 1979, 33, 555-561.

[36.] V.P. Tomaselli, R. Rivera, D.C. Edewaard and K.D. Möller, Appl. Opt., 1981, 20, 3961-3967.

[37.] C.G. Granqvist and O. Hunderi, Phys. Rev. B, 1977, 16, 3513-3534.

[38.] J. A. Stratton: Electromagnetic Theory, New York: McGraw-Hill, 1941. 


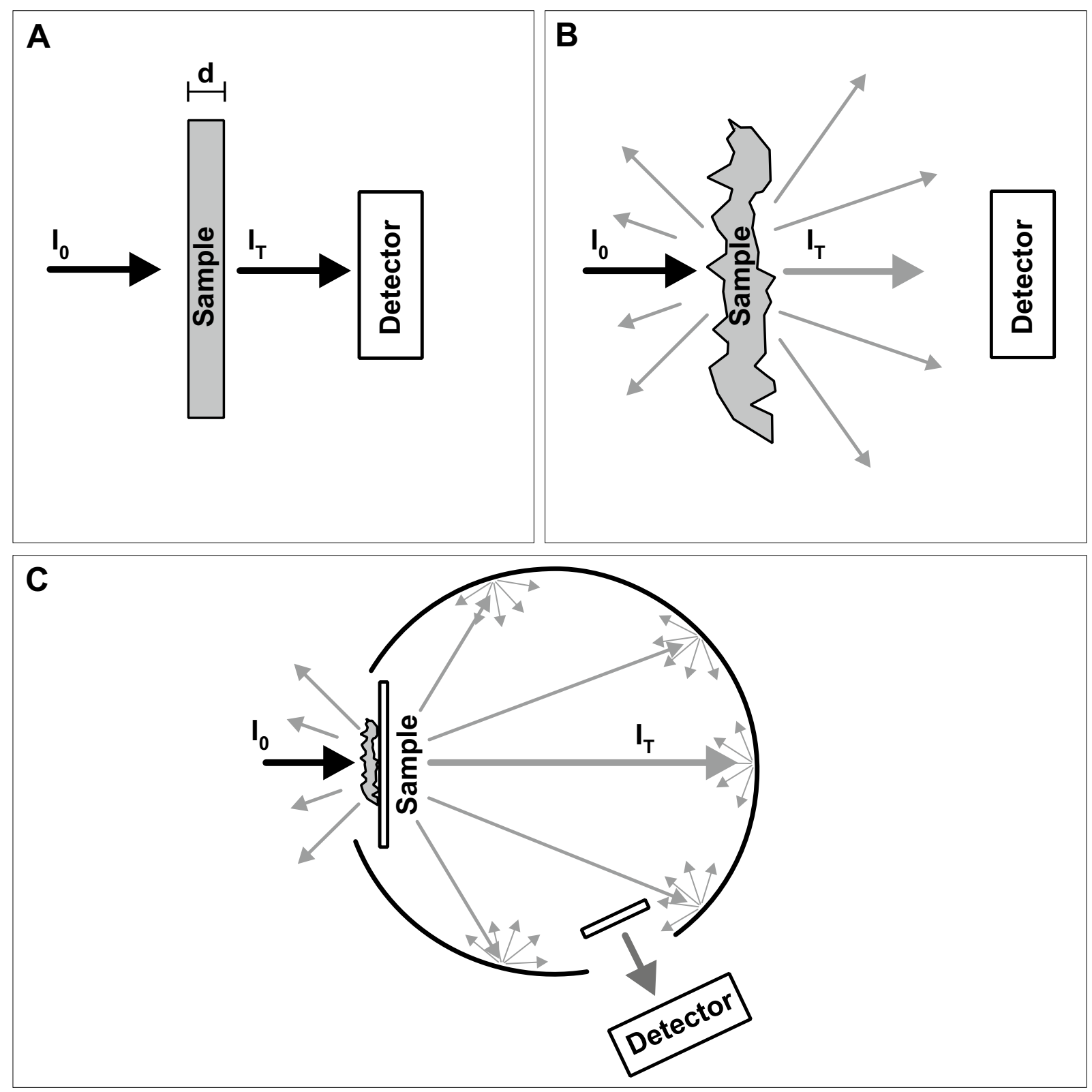

Dazzi et al., Fig. 01, vers. February 21, 2013 


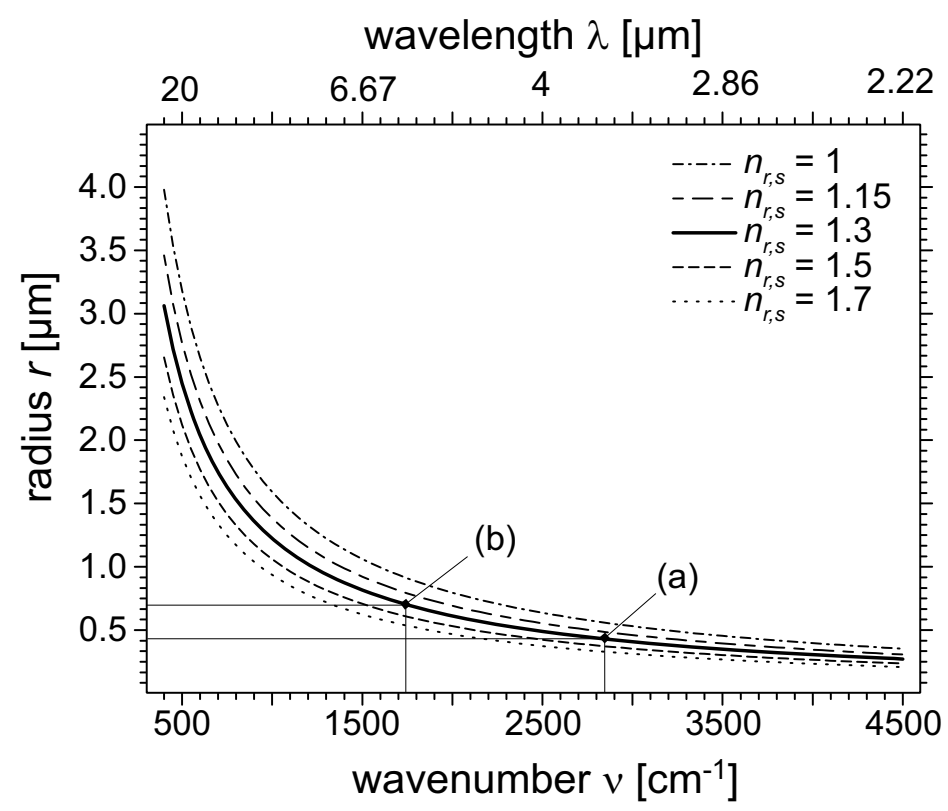

Dazzi et al., Fig. 02, vers. February 21, 2013 


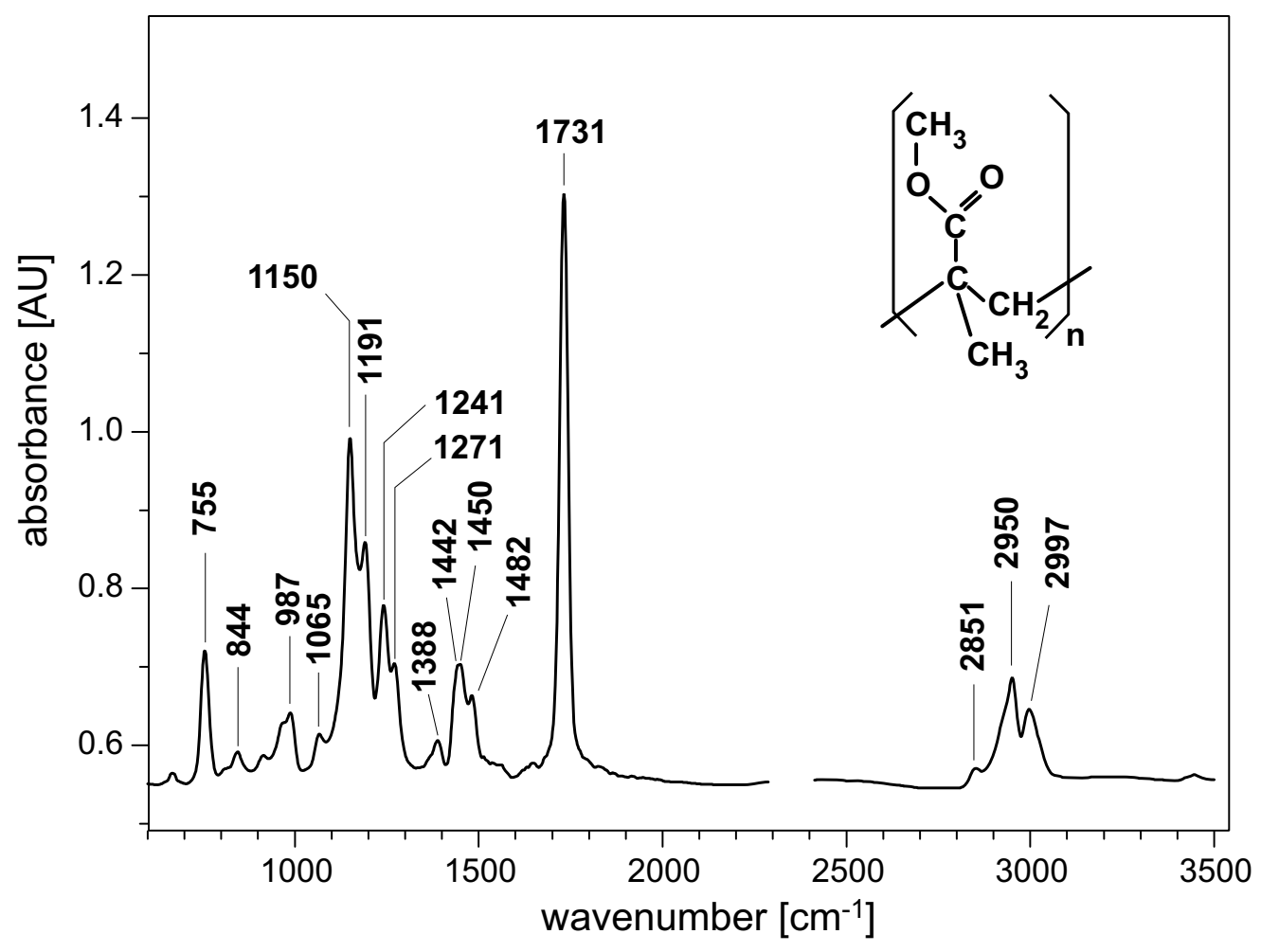

Dazzi et al., Fig. 03, vers. February 21, 2013 

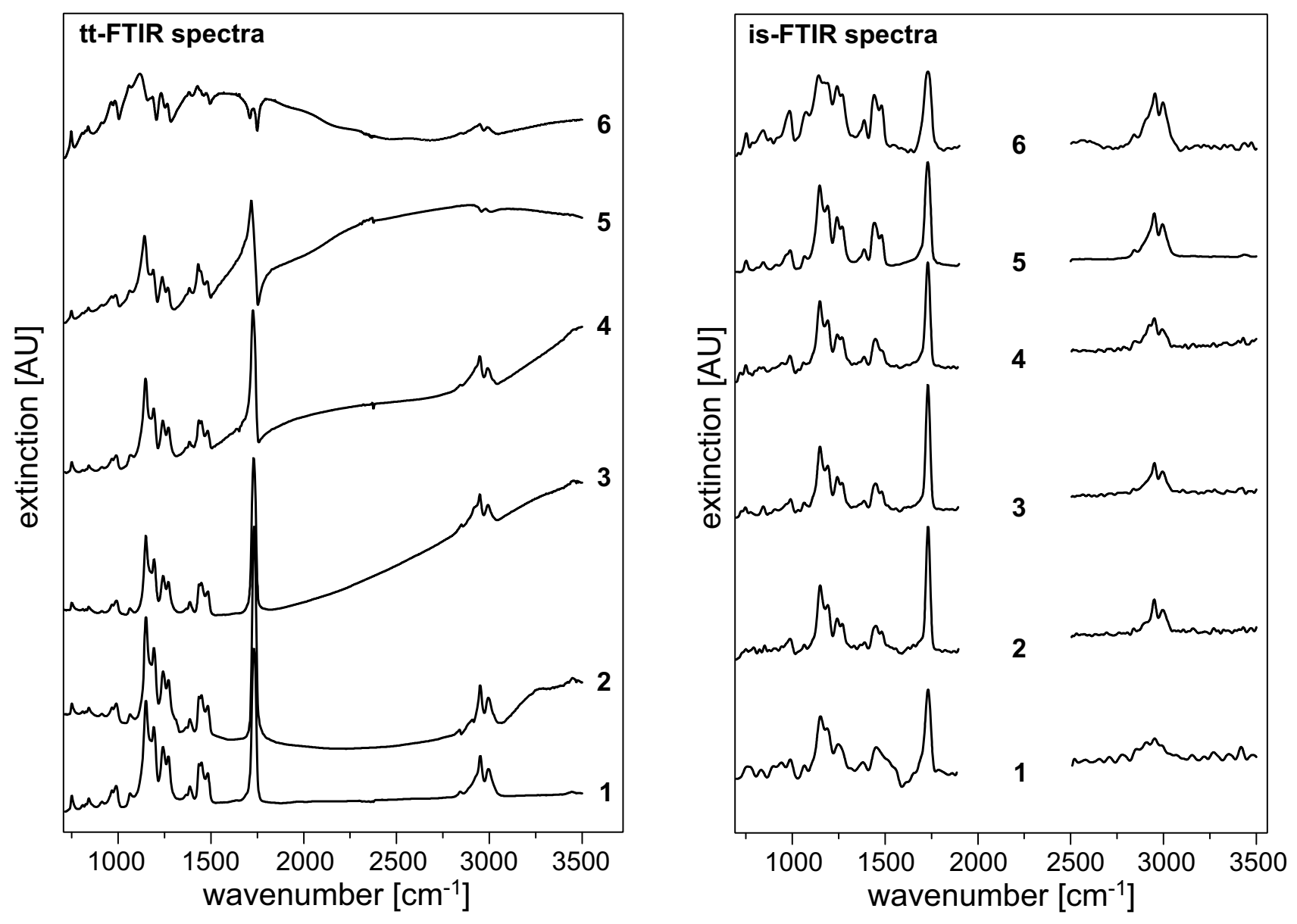

Dazzi et al., Fig. 04, vers. February 21, 2013 


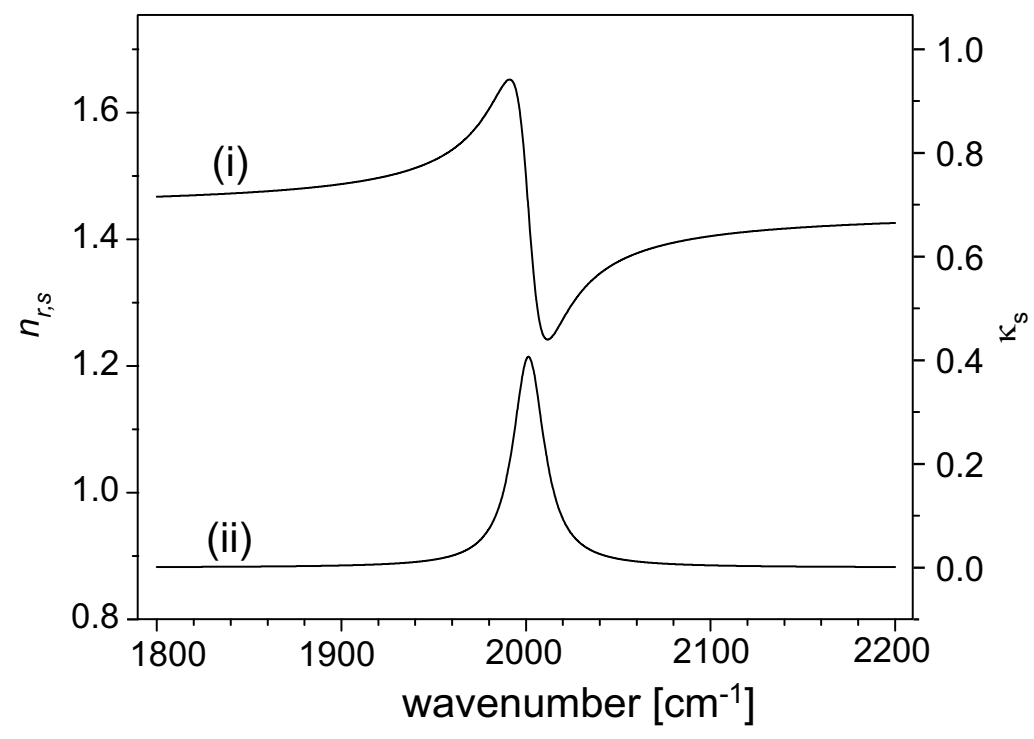

Dazzi et al., Fig. A, vers. February 21, 2013 


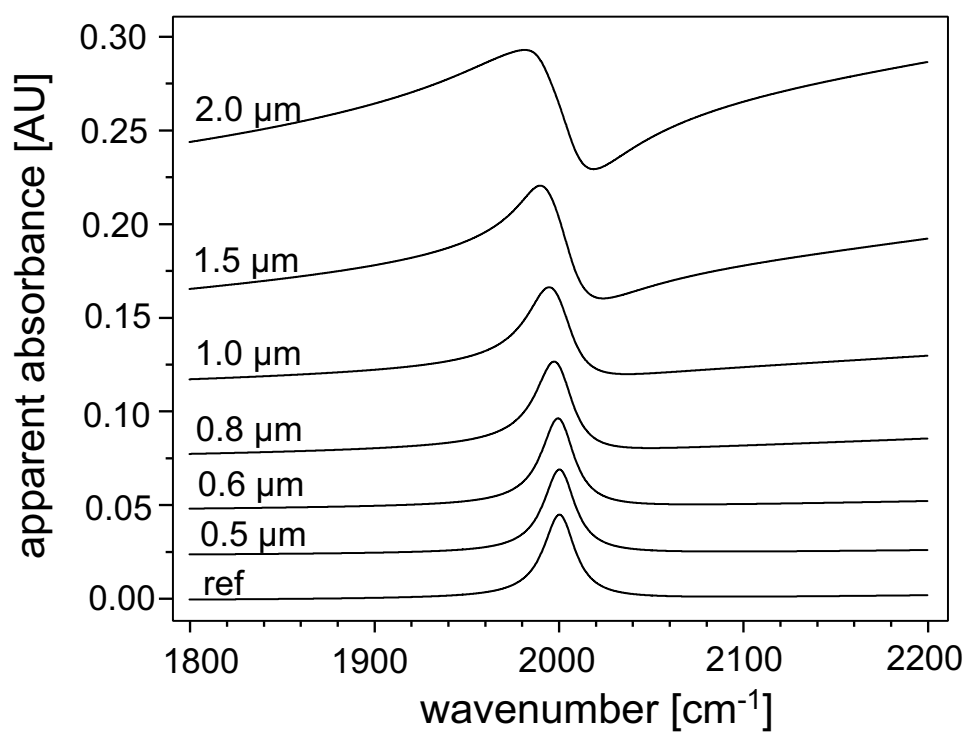

Dazzi et al., Fig. B, vers. April 24, 2013 
Supplementary Material (ESI) for Analyst

Electronic supplementary information (ESI)

\title{
Minimising Contributions from Scattering in Infrared Spectra by Means of an Integrating Sphere
}

\author{
Alexandre Dazzi ${ }^{1}$, Ariane Deniset ${ }^{1}$, Peter Lasch ${ }^{2, \$}$ \\ ${ }^{1}$ Laboratoire de Chimie Physique, UMR8000, Université Paris-Sud 11, 91405 Orsay, France. \\ ${ }^{2}$ Robert Koch-Institute, Centre for Biological Security 6 (ZBS 6), Nordufer 20, 13353 Berlin, \\ Germany \\ \$ corresponding author, e-mail: LaschP@rki.de
}



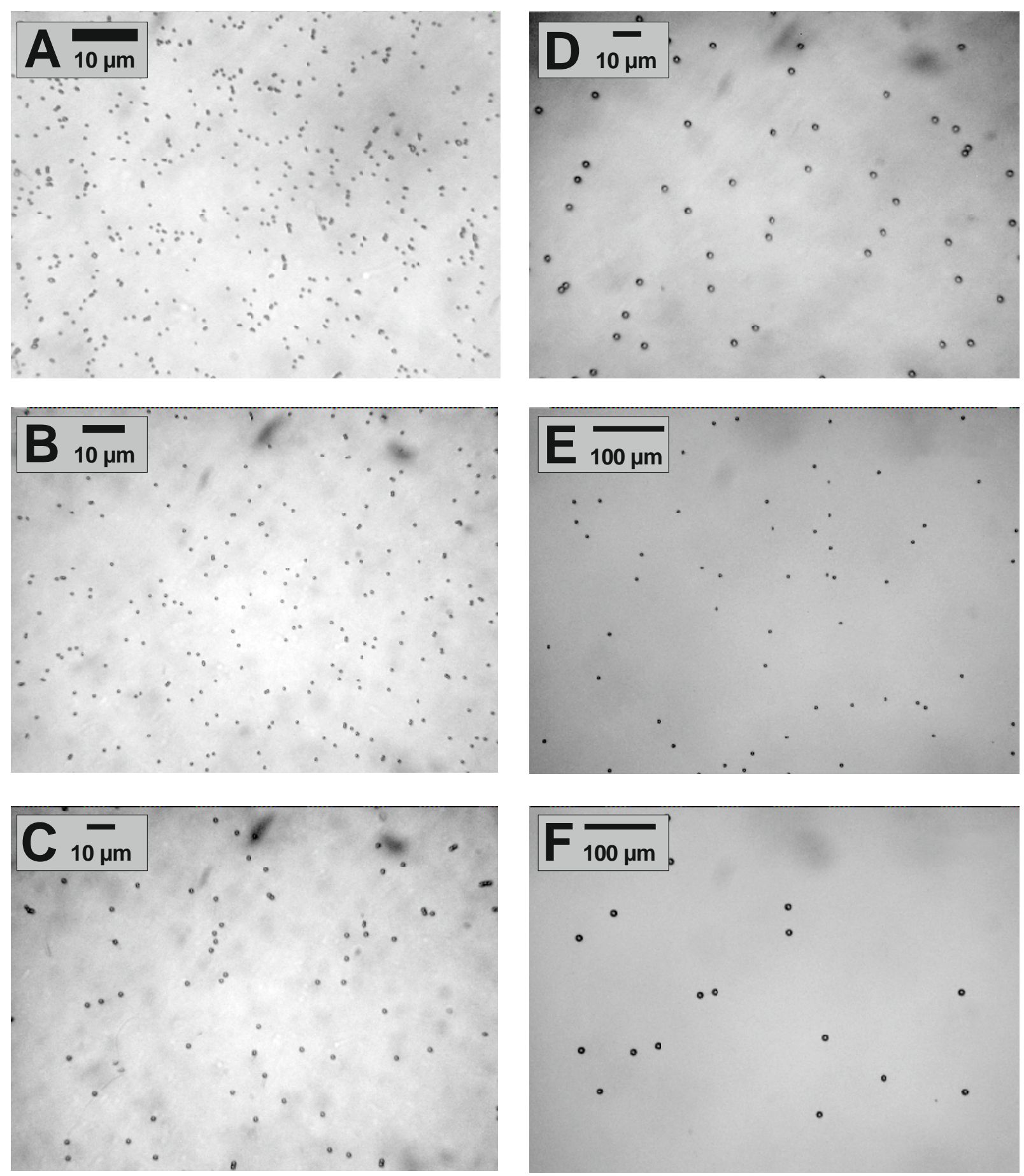

\section{Figure S1}

Light microscopy images of the PMMA microsphere preparations on ZnSe windows. These photographs demonstrate the absence of stacking and illustrate that contacts between individual microspheres can be considered an exception.

Radii $r$ of the PMMA spheres:

A.) $r=0.242 \mu$, B.) $r=0.404 \mu \mathrm{m}$, C.) $r=0.62 \mu \mathrm{m}$, D.) $r=1.04 \mu \mathrm{m}$, E.) $r=2.31 \mu \mathrm{m}$, F.) $r=$ $4.86 \mu \mathrm{m}$. 